\title{
Photoproduction in Ultra-Peripheral Heavy Ion Collisions at STAR
}

\author{
B. Grube ${ }^{\mathrm{a} b}$ for the STAR Collaboration

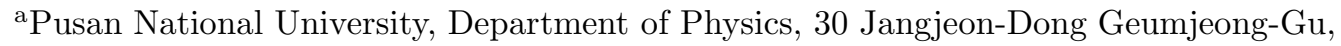 \\ Busan 609-735, Republic of Korea

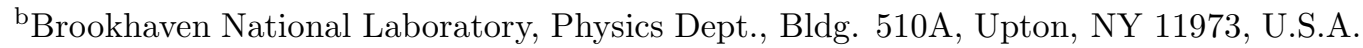

We present recent STAR results on photoproduction in ultra-peripheral relativistic heavy ion collisions. In these collisions the impact parameter of the beam particles is larger than the sum of their nuclear radii, so that they interact via their long-range Coulomb fields. STAR has measured the production of $\rho^{0}(770)$ mesons in exclusive reactions as well as in processes with mutual nuclear excitation of the beam particles. We present results for the $\rho^{0}$ production cross section in $\mathrm{Au}-\mathrm{Au}$ collisions at $\sqrt{s_{N N}}=200 \mathrm{GeV}$ for coherent as well as incoherent coupling. The dependence of the cross section on the $\rho^{0}$ rapidity is compared to theoretical models. We also studied the ratio of coherent $\rho^{0}$ to direct $\pi^{+} \pi^{-}$production as well as the $\rho^{0}$ helicity matrix elements and we observe interference effects in the $\rho^{0}$ production. In addition STAR has measured the production of $\rho^{0}$ mesons in $d$-Au collisions at $\sqrt{s_{N N}}=200 \mathrm{GeV}$ and that of $e^{+} e^{-}$-pairs in $\mathrm{Au}-\mathrm{Au}$ at $\sqrt{s_{N N}}=200 \mathrm{GeV}$. We also see an enhancement around $1510 \mathrm{MeV} / \mathrm{c}^{2}$ in $\pi^{+} \pi^{-} \pi^{+} \pi^{-}$final states in Au-Au collisions at $\sqrt{s_{N N}}=200 \mathrm{GeV}$.

\section{Introduction}

Heavy ions are surrounded by a cloud of virtual photons. The electromagnetic field of a relativistic nucleus can be approximated by a flux of quasi-real virtual photons using the WeizsäckerWilliams approach [1. Since the number of photons grows with the square of the nuclear charge, fast moving heavy ions can generate large photon fluxes. Beams of heavy ions can thus be used as photon sources or targets. Due to the long range of the electromagnetic interactions, they can be separated from the hadronic interactions by requiring impact parameters $b$ larger than the sum of the nuclear radii $R_{A}$ of the beam particles. These so-called Ultra-Peripheral heavy ion Collisions (UPCs) allow to study photonuclear effects as well as photon-photon interactions [2].

A typical photonuclear reaction is the production of vector mesons. In this process the virtual photon, that is emitted by the "spectator" nucleus, fluctuates into a virtual $q \bar{q}$-pair, which scatters elastically off the "target" nucleus, thus producing a real vector meson. The scattering can be described in terms of soft Pomeron exchange. Figure 1h illustrates this reaction taking as an example the most abundant process, the photonuclear production of $\rho^{0}(770)$ mesons.

The cross section for vector meson production depends on the way the virtual $q \bar{q}$-pair couples to the target nucleus. This is mainly determined by the transverse momentum $p_{T}$ of the produced meson. For small transverse momenta of the order of $p_{T} \lesssim 2 \hbar / R_{A}$ the $q \bar{q}$-pair couples coherently to the entire nucleus. This leads to large cross sections which depend on the nuclear form factor $F(t)$ and which scale roughly with the atomic number $A$ squared. For larger transverse momenta the $q \bar{q}-$ pair couples to the individual nucleons in the target nucleus. This "incoherent" scattering has a smaller cross section that scales approximately with $A$ modulo corrections for nuclear absorption of the meson.

Due to the intense photon flux, it is possible for the vector meson production to be accompanied by Coulomb excitation of the beam particles. The excited ions mostly decay via the emission of neutrons which can be measured by the Zero Degree Calorimeters (ZDCs) [3] of the STAR setup. To lowest order events with mutual nuclear breakup are described by three-photon 


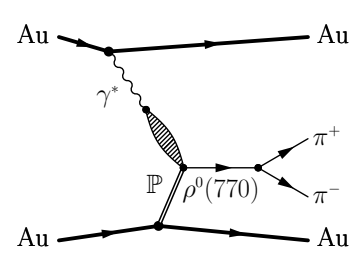

a)

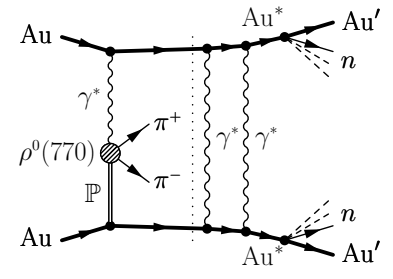

b)
Figure 1. (a) Exclusive photonuclear $\rho^{0}$ production in $\mathrm{Au}-\mathrm{Au}$ ultra-peripheral collisions. (b) Photonuclear $\rho^{0}$ production accompanied by mutual Coulomb excitation of the ions. The $\rho^{0}$ creation in $\gamma^{*} \mathbb{P}$ fusion is adumbrated in form of a hatched circle.

exchange (see Fig. 10): One photon to produce the vector meson and two photons to excite the two nuclei [4. All three photon exchanges are in good approximation independent, so that the cross section for the production of a vector meson $V$ can be factorized:

$$
\begin{aligned}
& \sigma\left(\mathrm{AuAu} \rightarrow \mathrm{Au}^{*} \mathrm{Au}^{*} V\right)= \\
& \int \mathrm{d}^{2} b\left[1-P_{\mathrm{had}}(b)\right] P_{V}(b) P_{X n, 1}(b) P_{X n, 2}(b)
\end{aligned}
$$

where $P_{\text {had }}$ is the probability for hadronic interaction, $P_{V}$ the probability to produce a vector meson $V$, and $P_{X n, i}$ the probability that nucleus $i$ emits $X$ neutrons. Compared to exclusive vector meson production, reactions with mutual Coulomb excitation have smaller median impact parameters.

In this paper we present results from the STAR experiment at the Relativistic Heavy Ion Collider (RHIC). Charged tracks were reconstructed using a large cylindrical Time Projection Chamber (TPC) [5] with $2 \mathrm{~m}$ radius and a length of $4.2 \mathrm{~m}$, that was operated in a $0.5 \mathrm{~T}$ solenoidal magnetic field. For tracks with pseudorapidity $|\eta|<1.2$ and transverse momentum $p_{T}>$ $100 \mathrm{MeV} / c$ the tracking efficiency is better than $85 \%$. For triggering two detector systems were used: the Central Trigger Barrel (CTB) [6], which is an array of 240 scintillator slats surrounding the TPC that allows to trigger on charged par- ticle multiplicities in the event, and the two zero degree calorimeters, which are located at $\pm 18 \mathrm{~m}$ from the interaction point and measure neutrons originating from nuclear breakup.

\section{Photonuclear $\rho^{0}$ Production}

STAR uses two kinds of triggers to select UPC events. The CTB-based "topology" trigger requires a low total charged particle multiplicity and divides the CTB detector into four azimuthal quadrants. Events are recorded when the left and right quadrants have coincident hits, selecting roughly back-to-back pion pairs. The top and bottom quadrants serve as a cosmic ray veto. The "minimum bias" trigger selects events where both nuclei dissociated by requiring coincident energy deposits in both ZDCs.

In the offline analysis the events were required to have two tracks of opposite charge that form a common (primary) vertex. In addition we demanded a low overall track multiplicity in order to suppress backgrounds from beam-gas interactions, peripheral hadronic interactions, and pileup events. The primary vertex was confined to a region close to the interaction diamond which reduces contaminations from pile-up events, beamgas interactions, and cosmic rays. The latter background component was decreased further by either excluding events around $y_{\rho}=0$ in the topology data sample or by requiring neutron signals in the ZDCs as in the minimum bias trigger. The contamination from peripheral hadronic interactions was in addition suppressed by demanding the track pair to have low net transverse momentum. The backgrounds from two-photon $e^{+} e^{-}$(cf. section 3 ) and photonuclear $\omega$ production are negligible.

The $\pi^{+} \pi^{-}$invariant mass distributions are fit with the function

$$
\begin{aligned}
& \frac{\mathrm{d} \sigma}{\mathrm{d} M_{\pi \pi}}=\left|A \frac{\sqrt{M_{\pi \pi} M_{\rho} \Gamma}}{M_{\pi \pi}^{2}-M_{\rho}^{2}+i M_{\rho} \Gamma}+B\right|^{2}+f_{\mathrm{BG}} \\
& \text { with } \Gamma\left(M_{\pi \pi}\right) \equiv \Gamma_{\rho} \frac{M_{\rho}}{M_{\pi \pi}}\left[\frac{M_{\pi \pi}^{2}-4 m_{\pi}^{2}}{M_{\rho}^{2}-4 m_{\pi}^{2}}\right]^{\frac{3}{2}}
\end{aligned}
$$

Equation (2) consists of a relativistic BreitWigner with amplitude $A$, that describes the res- 
onant $\pi^{+} \pi^{-}$production, and a mass-independent amplitude $B$ for the non-resonant $\pi^{+} \pi^{-}$production, as well as a Söding interference term [7] of the two amplitudes. The second order polynomial, $f_{\mathrm{BG}}$, describes the combinatorial background which was estimated from like sign pion pairs.

\subsection{Coherent $\rho^{0}$ Production in $\mathrm{Au}-\mathrm{Au}$ Collisions}

Coherent photonuclear $\rho^{0}$ production is strongly enhanced at low $p_{T}^{\rho}$. It can be separated from the incoherent production by requiring $\rho^{0}$ transverse momenta smaller than $150 \mathrm{MeV} / c$. Figure 2 shows the $\pi^{+} \pi^{-}$invariant mass distribution of the minimum bias data set taken during the year 2002 run in $\mathrm{Au}-\mathrm{Au}$ collisions at $\sqrt{s_{N N}}=200 \mathrm{GeV}[8$. The peak contains $3075 \pm 128_{\text {stat. }} \rho^{0}$ candidates.

Since in Eq. (2) the amplitudes $A$ and $B$ are free fit parameters, it is possible to extract the ratio $|B / A|$ of non-resonant to resonant $\pi^{+} \pi^{-}$ production. In $\mathrm{Au}-\mathrm{Au}$ collisions at $\sqrt{s_{N N}}=$ $200 \mathrm{GeV}$ the ratio was measured using the minimum bias data to $|B / A|=0.89 \pm 0.08_{\text {stat. }} \pm$ $0.09_{\text {syst. }} \mathrm{GeV}^{-1 / 2}[$ [] . As expected the ratio shows no dependence on the $\rho^{0}$ rapidity nor on the $\rho^{0}$ decay angles. The value is compatible with an earlier result of $|B / A|=0.81 \pm 0.08_{\text {stat. }} \pm$ $0.20_{\text {syst. }} \mathrm{GeV}^{-1 / 2}$ from the year 2000 run at $\sqrt{s_{N N}}=130 \mathrm{GeV}$ [9]. Both values are also in agreement with the ZEUS measurements [10].

The luminosity of the minimum bias sample was measured based on the known total hadronic production cross section of 7.2 barn. With the value of $L_{\text {minbias }}=461 \mathrm{mb}^{-1}$ the cross section for coherent $\rho^{0}$ production accompanied by mutual nuclear excitation was estimated to be $14.5 \pm 0.7_{\text {stat. }} \pm 1.9_{\text {syst. }} \mathrm{mb}^{-1}$ within the experimental acceptance of $\left|y_{\rho}\right|<1[8$.

There are at least three models for $\rho^{0}$ production in ultra-peripheral collisions: The model of Klein and Nystrand (KN) [1] uses the Vector Dominance Model (VDM) to describe the virtual photon and a classical mechanical approach for the scattering on the target nucleus, based on results from $\gamma p \rightarrow \rho^{0} p$ experiments. The Frankfurt, Strikman, and Zhalov (FSZ) model [12] em-

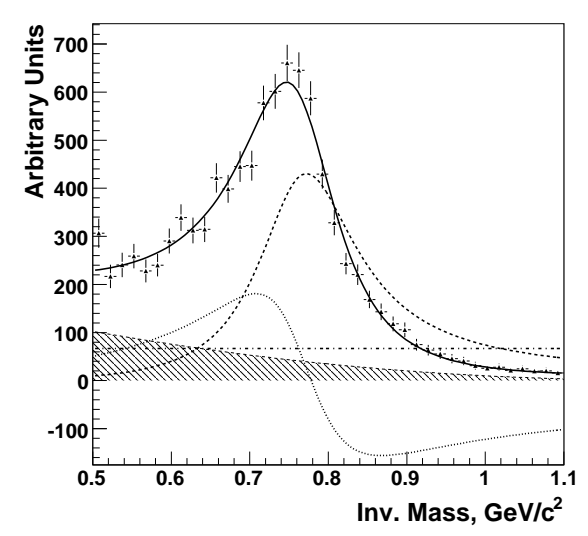

Figure 2. $\pi^{+} \pi^{-}$invariant mass distribution for coherently produced $\rho^{0}$ from the minimum bias data set [8]. The solid line is the fit result using Eq. (2), which has four components: The relativistic Breit-Wigner of the resonant $\pi^{+} \pi^{-}$production (dashed line), the mass-independent term for the non-resonant $\pi^{+} \pi^{-}$production (dashdotted line), the interference of the two (dotted line), and the combinatorial background (hatched area).

ploys a generalized VDM for the virtual photon and a QCD Gribov-Glauber approach for the scattering. The model of Gonçalves and Machado (GM) [13] is based on a QCD color dipole approach that takes into account nuclear effects and parton saturation phenomena. Only the KN model gives predictions for the rapidity dependence of the coherent $\rho^{0}$ production cross section accompanied by mutual Coulomb excitation, which are compared to the data in Fig. 3 .

The total coherent $\rho^{0}$ production cross section was estimated using the $13054 \pm 124_{\text {stat. }} \rho^{0}$ candidates in the data set taken with the topology trigger [8], which does not apply any restrictions on the ZDC amplitudes. Although this data sample could not be used for an absolute cross section measurement, because the trigger efficiency is only poorly known, it was possible to extract coherent cross section ratios for the different nuclear excitation states. These ratios were used to 


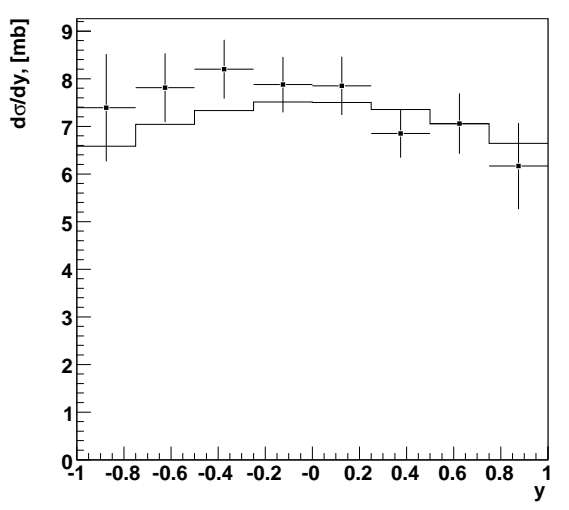

Figure 3. Differential cross section $\mathrm{d} \sigma / \mathrm{d} y_{\rho}$ for coherent $\rho^{0}$ production accompanied by mutual Coulomb excitation as a function of $\rho^{0}$ rapidity extracted from the minimum bias data [8]. The solid line shows the KN model calculation [11.

scale the coherent mutual-excitation cross section values from the minimum bias sample. Figure 4 compares the obtained $y_{\rho}$-dependence of the coherent $\rho^{0}$ production cross section to the three models. Unfortunately the limited acceptance in $y_{\rho}$ does not allow to distinguish between different shapes of the distributions.

STAR has also measured the spin structure of the coherent $\rho^{0}$ production amplitudes in terms of spin density matrix elements [8]. The matrix elements were extracted from the minimum bias data by fitting the $\rho^{0}$ decay angular distribution in the $\rho^{0}$ rest frame to the theoretical prediction for unpolarized photoproduction 14.

$$
\begin{aligned}
\frac{1}{\sigma} \frac{\mathrm{d}^{2} \sigma}{\mathrm{d} \cos \theta \mathrm{d} \phi}=\frac{3}{4 \pi}\left[\frac{1}{2}\left(1-r_{00}^{04}\right)\right. & \\
+\frac{1}{2}\left(3 r_{00}^{04}-1\right) \cos ^{2} \theta- & \sqrt{2} \mathfrak{R e}\left[r_{10}^{04}\right] \sin 2 \theta \cos \phi \\
& \left.-r_{1-1}^{04} \sin ^{2} \theta \cos 2 \phi\right]
\end{aligned}
$$

where $\theta$ is the polar angle between the beam direction and the direction of the $\pi^{+}$and $\phi$ the azimuthal angle between the decay and the production plane. Since STAR does not measure

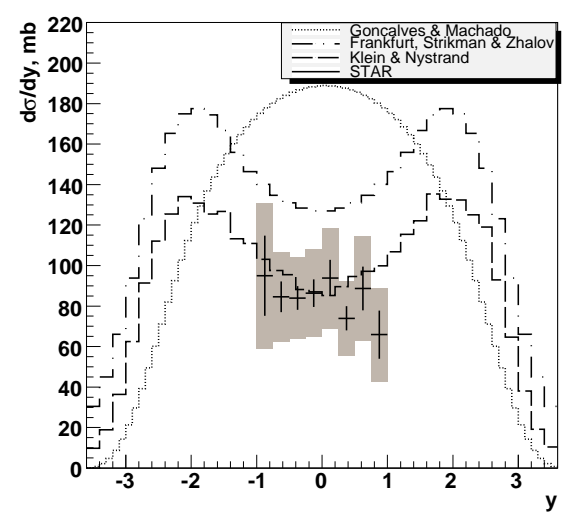

Figure 4. Comparison of the measured cross section for coherent $\rho^{0}$ production with theoretical predictions [8]. The vertical line at each point shows the statistical error. The shaded area displays the sum of statistical and systematical errors. The dashed line represents the KN [11, the dash-dotted line the FSZ [12], and the dotted one the GM model 13 .

the scattered beam particles, only an approximate production plane with respect to the beam direction could be reconstructed. $r_{00}^{04}$ represents the probability that the $\rho^{0}$ is produced with helicity $0, r_{1-1}^{04}$ the interference between helicity non-flip and double-flip. Due to the ambiguity of photon source and target, $\mathfrak{R e}\left[r_{10}^{04}\right]$, which is related to the interference between helicity nonflip and single-flip, could not be measured. Table 1 summarizes the results. The measured spin density elements are small, indicating $s$-channel helicity conservation. They are also compatible with ZEUS measurements on $\rho^{0}$ photoproduction from proton targets [10].

\subsection{Interference Effects in Coherent $\rho^{0}$ Production in Au-Au Collisions}

In ultra-peripheral collisions the impact parameter $b$ is typically much larger than the sum of the nuclear radii of the beam particles. The scattering of the virtual $q \bar{q}$-pair on the target nucleus, on the other hand, involves the short- 
Table 1

Spin density matrix elements for coherent $\rho^{0}$ production accompanied by mutual Coulomb excitation in $\mathrm{Au}$-Au collisions at $\sqrt{s_{N N}}=200 \mathrm{GeV}$ [8] compared to ZEUS results from $\rho^{0}$ production in $\gamma p$ collisions [10].

\begin{tabular}{rcr}
\hline Parameter & STAR & ZEUS \\
\hline$r_{00}^{04}$ & $-0.03 \pm 0.03_{\text {stat. }} \pm 0.06_{\text {syst. }}$ & $0.01 \pm 0.01_{\text {stat. }} \pm 0.02_{\text {syst. }}$ \\
$\mathfrak{R e}\left[r_{10}^{04}\right]$ & - & $0.01 \pm 0.01_{\text {stat. }} \pm 0.01_{\text {syst. }}$ \\
$r_{1-1}^{04}$ & $-0.01 \pm 0.03_{\text {stat. }} \pm 0.05_{\text {syst. }}$ & $-0.01 \pm 0.01_{\text {stat. }} \pm 0.01_{\text {syst. }}$ \\
\hline
\end{tabular}

ranged $(\mathcal{O}(1 \mathrm{fm}))$ strong interaction, so that the production of the $\rho^{0}$ mesons occurs in or very near to the target nucleus. Furthermore photon source and target are indistinguishable, so that either nucleus 1 emits a photon which produces a $\rho^{0}$ at nucleus 2 , or vice versa. The system thus behaves like a two-source interferometer with separation $b$, where the interference between the two possible amplitudes creates an entangled final state $\pi^{+} \pi^{-}$ wave function. Since the $\rho^{0}$ has negative parity, the two amplitudes have different sign, so that the cross section can be written as 15 .

$\sigma(b)=\left|A\left(b, y_{\rho}\right)-A\left(b,-y_{\rho}\right) e^{i \vec{p}_{T}^{\rho} \cdot \vec{b}}\right|^{2}$

where $A\left(b, y_{\rho}\right)$ and $A\left(b,-y_{\rho}\right)$ are the $\rho^{0}$ production amplitudes for the two photon directions. The observed $p_{T}^{\rho}$ spectrum is obtained by integrating Eq. (4) over $\vec{b}$, which is not measured in the experiment. For $y_{\rho}=0$ the two amplitudes are equal so that

$\sigma(b)=\sigma_{0}(b)\left[1-\cos \left(\vec{p}_{T}^{\rho} \cdot \vec{b}\right)\right]$

with $\sigma_{0}(b)$ being the cross section without interference. From Eq. (5) it is clear that the cross section is suppressed for $p_{T}^{\rho} \lesssim 2 \hbar /\langle b\rangle$, where $\langle b\rangle$ is the mean impact parameter.

The $t$-spectra, with $t \approx\left(p_{T}^{\rho}\right)^{2}$, are parametrized by

$\frac{\mathrm{d} N}{\mathrm{~d} t}=A e^{-B t}[1+c(R(t)-1)]$

where $R(t) \equiv[\mathrm{d} N / \mathrm{d} t]_{\text {int }}^{\mathrm{MC}} /[\mathrm{d} N / \mathrm{d} t]_{\text {noint }}^{\mathrm{MC}}$ is the ratio of the $t$-distributions with and without interference obtained from Monte-Carlo simulations based on the KN model 11. $A$ is the overall normalization, $B$ the slope parameter for coherent $\rho^{0}$ production, and $c$ measures the strength of the interference: $c=0$ corresponds to no interference, whereas $c=1$ is the expected interference in the $\mathrm{KN}$ model.

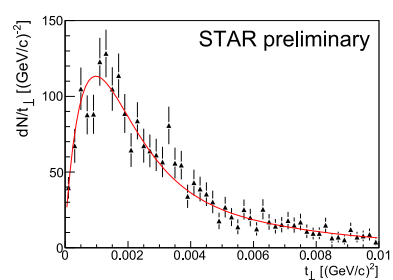

a)

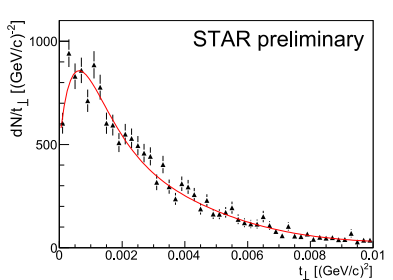

b)
Figure 5. Efficiency-corrected $t$-spectra for (a) the minimum bias data set with $\left|y_{\rho}\right|<0.5$ and for (b) the topology data set with $0.05<\left|y_{\rho}\right|<$ 0.5. The minimum bias data exhibit a wider dip at low $t$, because the median impact parameter is smaller. The solid line shows a fit to Eq. (6).

Due to the neutron tagging of mutual Coulomb excitation in the minimum bias trigger, the median impact parameter of $\tilde{b} \approx 18 \mathrm{fm}$ in this data set is smaller than the $\tilde{b} \approx 46 \mathrm{fm}$ in the topology data. Therefore the dip in the minimum bias $t$-spectra should extend to larger values of $t$. Figure 5 shows the efficiency-corrected $t$-distributions for the run 2002 topology and minimum bias data sets from $\mathrm{Au}-\mathrm{Au}$ collisions at $\sqrt{s_{N N}}=200 \mathrm{GeV}$ in the central rapidity region $\left|y_{\rho}\right|<0.5$ together with a fit to Eq. (6). To avoid contamination of the topology sample by cosmic 
rays, in addition the region $\left|y_{\rho}\right|<0.05$ was excluded.

The minimum bias data exhibit an interference strength of $c=0.92 \pm 0.07_{\text {stat. }}$ close to the expected value. The value from the topology data set is lower with $c=0.73 \pm 0.10_{\text {stat. }}$, but could be affected by the imperfect topology trigger simulation.

\subsection{Incoherent $\rho^{0}$ Production in $\mathrm{Au}-\mathrm{Au}$ Collisions}

The incoherent $\rho^{0}$ production, where the $\rho^{0}$ couples to the individual nucleons, can be measured by extending the analyzed range of the $\rho^{0}$ transverse momentum to $550 \mathrm{MeV} / c$. If the region of $t<0.002(\mathrm{GeV} / c)^{2}$, where interference effects reduce the cross section (see subsection 2.2), is excluded, the $t$-distribution can be described by a double exponential function

$\frac{\mathrm{d} \sigma}{\mathrm{d} t}=A_{\mathrm{coh}} e^{-B_{\mathrm{coh}} t}+A_{\mathrm{inc}} e^{-B_{\mathrm{inc}} t}$

$B_{\text {coh }}$ is the slope parameter of the exponential that dominates the low- $p_{T}^{\rho}$ region and corresponds to the coherent production. Accordingly $B_{\text {inc }}$ is the slope parameter of the incoherent production that extends to larger $p_{T}^{\rho}$.

Figure 66 shows a fit of Eq. (77) to the year 2002 minimum bias data from $\mathrm{Au}-\mathrm{Au}$ at $\sqrt{s_{N N}}=$ $200 \mathrm{GeV}$ that gives $B_{\text {coh }}=388 \pm 24_{\text {stat. }}(\mathrm{GeV} / c)^{-2}$ and $B_{\text {inc }}=8.8 \pm 1.0_{\text {stat. }}(\mathrm{GeV} / c)^{-2}[8]$. By integrating the exponentials the cross section ratio of incoherent to coherent $\rho^{0}$ production accompanied by mutual Coulomb excitation was measured to $29 \pm 3_{\text {stat. }} \pm 8_{\text {syst. }} \%$.

\section{4. $\rho^{0}$ Production in $d$-Au Collisions}

In $d$-Au ultra-peripheral collisions the virtual photon is predominantly emitted by the gold nucleus, due to its larger charge. There are two possible $\rho^{0}$ production processes: coherent scattering on the entire deuteron, $\gamma^{*} d \rightarrow \rho^{0} d$, and "incoherent" scattering on the individual nucleons of the deuteron in the course of which the deuteron dissociates, $\gamma^{*} d \rightarrow \rho^{0} p n$.

During the year 2003 run STAR took data of $d$-Au collisions at $\sqrt{s_{N N}}=200 \mathrm{GeV}$ using two UPC triggers. The first trigger was a topology

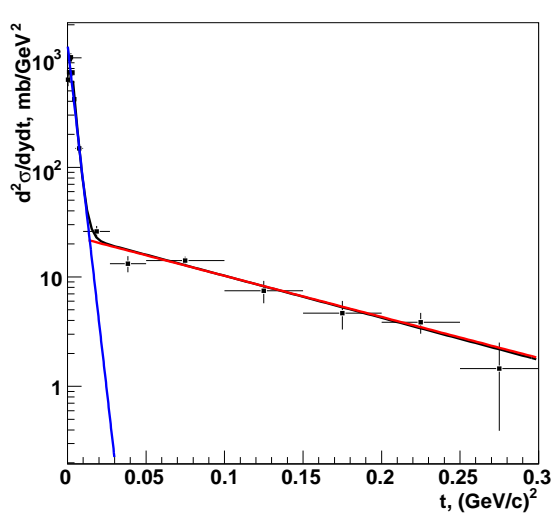

Figure 6. Dependence of the $\rho^{0}$ production cross section on the squared momentum transfer $t$ for the minimum bias data 8 . The solid line shows the result of a fit to the double exponential Eq. (7) with the steep line at low $t$ corresponding to coherent production. The line at larger $t$ describes the incoherent $\rho^{0}$ production.

trigger which selected mainly coherent interactions. The second trigger required, in addition to the topology condition, a neutron signal from the deuteron breakup in the corresponding ZDC. This data sample contains incoherent scattering events.

Figure 7 shows the $t$-spectrum, with $t \approx$ $\left(p_{T}^{\rho}\right)^{2}$, for incoherent scattering accompanied by deuteron dissociation. A fit with an exponential gives a slope parameter of $B=9.1 \pm$ $0.9_{\text {stat. }}(\mathrm{GeV} / \mathrm{c})^{-2}$ which is related to the nucleon form factor. This value is compatible with the slope parameter of $B_{\text {inc }}=8.8 \pm 1.0_{\text {stat. }}(\mathrm{GeV} / c)^{-2}$ measured in incoherent $\rho^{0}$ production accompanied by mutual Coulomb excitation in $\mathrm{Au}-\mathrm{Au}$ collisions at $\sqrt{s_{N N}}=200 \mathrm{GeV}$ (cf. subsection [2.3. 8]) and also agrees with the value of $B=10.9 \pm 0.3_{\text {stat. }}{ }_{-0.5}^{+1.0}$ syst. $(\mathrm{GeV} / c)^{-2}$ measured at ZEUS [10]. The downturn of the distribution at low $t$, which is also seen in low energy $\gamma d$ experiments [16], is due to the fact that there is not enough energy to dissociate the deuteron. 


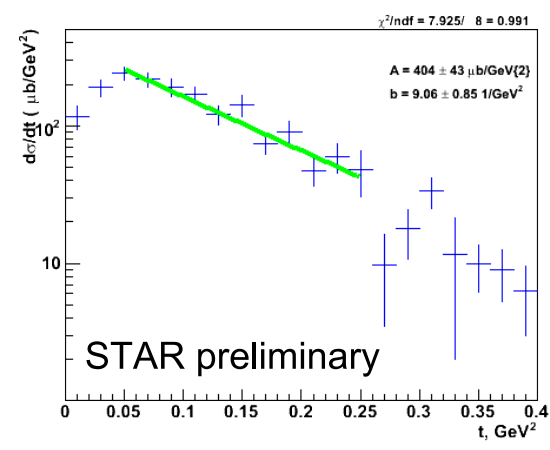

Figure 7. $t$-distribution for incoherent $\rho^{0}$ production accompanied by deuteron dissociation. The solid line shows an exponential fit.

\section{3. $e^{+} e^{-}$-Pair Production in Photon- Photon Interactions}

During the year 2002 run STAR also measured the production of $e^{+} e^{-}$-pairs in photon-photon interactions in ultra-peripheral $\mathrm{Au}-\mathrm{Au}$ collisions at $\sqrt{s_{N N}}=200 \mathrm{GeV}$ [17. Since the electromagnetic field is strong with $Z \alpha \approx 0.6$, one expects to see higher-order QED effects. In order to enrich collisions at small impact parameters, where the fields are strongest and thus higher-order corrections should be most pronounced, a ZDC trigger was employed, requiring mutual nuclear dissociation and thus limiting the impact parameter to $2 R_{A}<b \lesssim 30 \mathrm{fm}$.

In the offline analysis events were selected that had two reconstructed tracks of opposite charge emerging from a common (primary) vertex along the beamline. Up to two additional tracks, not associated to the primary vertex, were allowed in order to account for random backgrounds. Most of the $e^{ \pm}$are produced in forward direction with low transverse momentum, so that only a small fraction of the pairs reached the central detectors, even though the solenoid field was reduced to $0.25 \mathrm{~T}$, half of its nominal value. The tracks were required to have pseudorapidities of $|\eta|<1.15$ and transverse momenta of $65<p_{T}<130 \mathrm{MeV} / c$. The latter cut ensured that the $e^{ \pm}$identification via the specific energy loss in the TPC gas had only minimal contaminations from other particle species. Additional cuts on the pair transverse momentum, $p_{T}^{e^{+} e^{-}}<100 \mathrm{MeV} / c$, and the pair rapidity, $\left|y_{e^{+} e^{-}}\right|<1.15$, gave a final sample of 52 events in the pair invariant mass region of $140<M_{e^{+} e^{-}}<265 \mathrm{MeV} / c^{2}$.

The measured cross section in the selected kinematic range is $1.6 \pm 0.2_{\text {stat. }} \pm 0.3_{\text {syst. }}$ mb. Figure $8 \mathrm{a}$ shows the cross section as a function of the transverse momentum of the $e^{+} e^{-}$-pair, Fig. 8b the pair invariant mass distribution. The data are compared to a a recent QED calculation of Baltz that includes a realistic phenomenological treatment of the nuclear Coulomb excitation [18. The lowest order QED (dashed line in Fig. 8) overpredicts the data. The calculated cross section of $2.34 \mathrm{mb}$ is nearly 2 standard deviations above the data. The corresponding higher-order QED calculation gives a cross cestion value of $1.67 \mathrm{mb}$, compatible with the measurement, and also describes the kinematic distributions well.

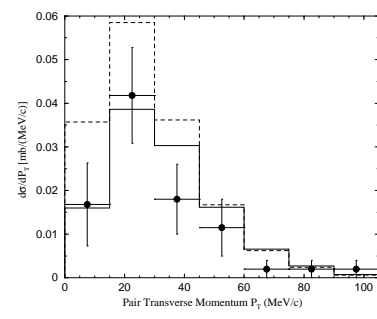

a)

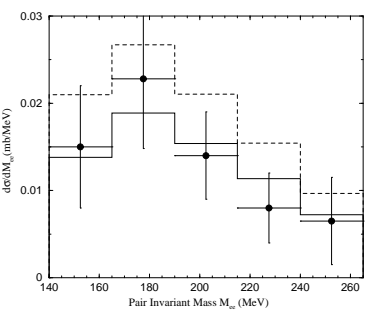

b)
Figure 8. (a) Pair transverse momentum spectrum. (b) $e^{+} e^{-}$invariant mass distribution. The dashed line shows the lowest order, the solid line the higher-order QED calculation according to 18 .

\section{Coherent Photonuclear $\pi^{+} \pi^{-} \pi^{+} \pi^{-}$ Production}

During the year 2004 run STAR took data in $\mathrm{Au}-\mathrm{Au}$ collisions at $\sqrt{s_{N N}}=200 \mathrm{GeV}$ using a "multi-prong" trigger which combined the UPC neutron tag in form of coincident ZDC signals 
with a low hit multiplicity in the CTB. In addition a veto from the large-tile Beam-Beam Counters (BBCs) [19], which cover $2.1<|\eta|<3.6$, was applied.

Events were selected that have four charged tracks coming from one common (primary) vertex. These four-prongs were required to be neutral and to have a net transverse momentum below $150 \mathrm{MeV} / c$, thus selecting coherent production. A low number of additional tracks, unassociated to the primary vertex, were allowed in the events to account for backgrounds.

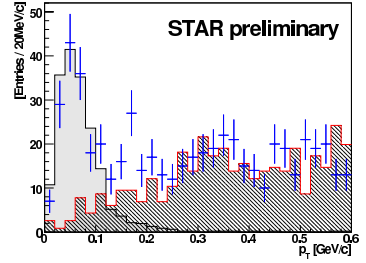

a)

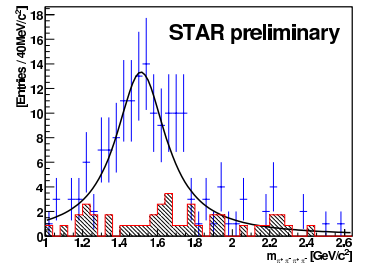

b)
Figure 9. (a) Transverse momentum spectrum of coherently produced neutral four-prongs. The points are the data, the shaded histogram depicts the expected distribution from simulation. The hatched histogram shows the background estimated from charged four-prongs. (b) $\pi^{+} \pi^{-} \pi^{+} \pi^{-}$ invariant mass distribution of coherently produced four-prongs. A broad peak indicates resonant $\pi^{+} \pi^{-} \pi^{+} \pi^{-}$production.

The transverse momentum distribution of the neutral four-prongs in Fig. 9a shows the expected peak at low $p_{T}$, characteristic for coherent production. The $\pi^{+} \pi^{-} \pi^{+} \pi^{-}$invariant mass spectrum in Fig. 9] shows a broad peak of approximately 100 events around $1510 \mathrm{MeV} / \mathrm{c}^{2}$ which could be caused by the production of excited $\rho^{0}$ states like the $\rho^{0}(1450)$ and/or the $\rho^{0}(1700)$.

\section{REFERENCES}

1. C. F. von Weizsäcker, Z. Phys. 88, 612 (1934); E. J. Williams, Phys. Rev. 45, 729 (1934).
2. G. Baur, K. Hencken, D. Trautmann, S. Sadovsky, and Yu. Kharlov, Phys. Rept. 364, 359 (2002); F. Krauss, M. Greiner, and G. Soff, Prog. Part. Nucl. Phys. 39, 503 (1997); C. A. Bertulani, S. R. Klein, and J. Nystrand, Ann. Rev. Nucl. Part. Sci. 55, 271 (2005); K. Hencken et al., Phys. Rept. 458, 1 (2008).

3. C. Adler et al., Nucl. Intrum. Methods A470, 488 (2001).

4. A. J. Baltz, S. R. Klein, and J. Nystrand, Phys. Rev. Lett. 89, 012301 (2002); G. Baur, K. Hencken, A. Aste, D. Trautmann, and S. R. Klein, Nucl. Phys. A729, 787 (2003).

5. M. Anderson et al., Nucl. Intrum. Methods A499, 659 (2003); ibid., at 679.

6. F. S. Bieser et al., Nucl. Intrum. Methods A499, 766 (2003).

7. P. Söding, Phys. Lett. 19, 702 (1966); J. J. Sakurai, Ann. Phys. 11, 1 (1960); T. H. Bauer et al., Rev. Mod. Phys. 50, 261 (1978).

8. B. I. Abelev et al., Phys. Rev. C77, 034910 (2008).

9. C. Adler et al., Phys. Rev. Lett. 89, 272302 (2002).

10. J. Breitweg et al., Eur. Phys. J. C2, 247 (1998).

11. S. Klein and J. Nystrand, Phys. Rev. C60, 014903 (1999).

12. M. Frankfurt, M. Strikman and M. Zhalov, Phys. Lett. B537, 51 (2002); ibid., Phys. Rev. C67, 034901 (2003).

13. V. P. Gonçalves and M. V. T. Machado, Eur. Phys. J. C40, 519 (2005).

14. K. Schilling et al., Nucl. Phys. B15, 397 (1970); K. Schilling and G. Wolf, Nucl. Phys. B61, 381 (1973).

15. S. R. Klein and J. Nystrand, Phys. Rev. Lett. 84, 2330 (2000); ibid., Phys. Lett. A308, 323 (2003)

16. Y. Eisenberg et al., Nucl. Phys. B104, 61 (1976).

17. J. Adams et al., Phys. Rev. C70, 031902(R) (2004).

18. A. J. Baltz, Phys. Rev. Lett. 100, 062302 (2008).

19. J. Kiryluk, 16th International Spin Physics Symposium Proc., hep-ex/0501072 (2004) 